\title{
Determinación de atributos y evaluación de la sustentabilidad de parcelas agrícolas (fincas) en la cuenca media y baja del río Supe, Barranca
}

Determination of attributes and sustainability assessment of agricultural ranchs (farms) in the middle and lower basin of the river Supe, Barranca

LOREnZO Ayora Garagate ${ }^{1}$

\section{RESUMEN}

En esta investigación se identificó los atributos socioeconómicos y técnico-productivos de fincas agrícolas con cultivos prevalentes; a continuación se evaluó la sustentabilidad utilizando indicadores ecológicos, económicos y socioculturales, en la cuenca baja y media del río Supe. Se caracterizó los sistemas prediales; luego se aplicó encuestas acordes con el diseño muestral; se efectuó la sistematización y los cálculos estadísticos. Finalmente se evaluó la sustentabilidad de las fincas con cultivos prevalentes, utilizando el método multicriterio y evaluación rápida de calidad del suelo. Los cultivos prevalentes son: maíz amarillo duro, caña de azúcar, palto, maracuyá, maíz morado y ají páprika. Los indicadores socioeconómicos encontrados determinan el estado de pobreza del agricultor y su familia; los principales sistemas de producción son los semiintensivos para la venta, la agricultura de subsistencia y la crianza menor extensiva. El índice de sustentabilidad para los cultivos prevalentes es variado, logrando sustentabilidad los cultivos de maíz amarillo duro, caña de azúcar y palto. Evaluada la calidad del suelo, se determinó valores que superan el umbral mínimo $(5,0)$ a excepción del ají páprika.

La determinación de los atributos técnico-productivos, socioeconómicos y culturales permitió evaluar la sustentabilidad, encontrándose que las fincas con palto, maíz amarillo duro y caña de azúcar alcanzan sustentabilidad.

Palabras clave: indicadores de sustentabilidad; sistemas agrícolas; análisis multicriterio; fincas.

\section{ABSTRACT}

In this research the attributes, socioeconomic and technical-productive farms with crops prevalent identified; then sustainability was evaluated using ecological, economic and socio-cultural indicators, in the middle of the river Supe and lower basin. The Building Systems was characterized, then according to the survey sample design was

1 Universidad Nacional «Santiago Antúnez de Mayolo». Huaraz, Perú. 
applied; systematizing and statistical calculations were made. Finally the sustainability of farms prevalent crops was assessed using the multi-criteria method and rapid assessment of soil quality. The prevalent crops are yellow corn, sugar cane, avocado, passion fruit, purple corn and paprika peppers. The found socioeconomic indicators determine the state of poverty of the farmer and his family; the main production systems are semi intensive for sale, subsistence agriculture, and less extensive breeding. The sustainability index for crops prevalent is varied, achieving sustainability crop yellow corn, sugar cane and avocado. Evaluated soil quality, values that exceed the minimum threshold (5.0) except paprika peppers was determined.

The determination of production, socioeconomic, cultural and technical attributes; allowed assessing sustainability, finding that farms with avocado, yellow corn, sugar cane and achieve sustainability.

Keywords: sustainability indicators; farming systems; multi-criteria analysis

\section{ICHIKLLACHAW}

Kay yachay ashiychaw tarikashqa alli murukuykunapa kaqninkunata, imanaw markantsikunachaw tanqanakushqanta; tsaytam rikapashqa kashqa imanawpis patsantsikchaw, qillayninchaw, kawaynintsikchawpis allikush mana allikush kashqa, tsay rurakashqa Supe mayupa kuchunkunachawmi. Tsaypaq imanawpis chakrakuna kashqantam rikapashqa kashqa, niykur rikaatsin nishqannaw tapukuyta rurakashqa, tsaytanam istadistika nishqanwan chanintsaatsiyarquu. Tsaykunawannam chakrakunata chanintsaayarquu. Atskata murukkuykuna kaynaw: qallwash hara, mishkip wiru, palto, marakuyaa, kulli hara, paprika utsupis. rikaatsin hina willakun atska marka nunakunam waktsa kayan; murukuyllachawtsu uryayay, murukuyan kawayaananllapaqmi, niykur waatayan ichik ashmakunata mikuyaananllapaqmi.

Alli murukuyqa manam llapan nunakunapaq tsaynawllatsu, qallwash hara, mishkip wiruwan paprika utsullam alli murukuy. Chakrakunata chanintsashqa willakun paprika utsulla mana chatsu umbral mínimuman $(5,0)$.

Imanawpis murukuy, kapamashqantsikkunawan imanawpis kawashqantsikwanmi chanintsashqa sustentabilidad nishqanta, tsaychawmi nin paltuwan, qallwash hara, mishkip wiruwan kaqkunallam tariyan tsay sustentabilidadta.

Pushaq shimikuna: alli murukuypa willakuqninkuna; tukuy murupakuy; tukuy laayapa rikaanan.

\section{INTRODUCCIÓN}

La investigación está orientada a determinar los atributos socioeconómicos y productivos de las parcelas agrícolas de la cuenca media y baja del río Supe, para evaluar y medir la sustentabilidad mediante el uso de indicadores de sustentabilidad, el análisis multicriterio y el análisis de calidad del suelo.

La trascendencia de este trabajo radica fundamentalmente en que, al término, se tendrá identificados los sistemas agrícolas más representativos de la zona en estudio; se habrá 
evaluado la sustentabilidad de las parcelas agrícolas con cultivos prevalentes y construido una base de datos con ubicación geoespacial que permitirá consultar y manejar información clasificada.

Para propender al uso racional, oportuno y sostenible de los recursos naturales y socioeconómicos de la cuenca media y baja del río Supe se hace necesario el conocimiento y análisis del territorio, sus limitaciones y potencialidades, y de una evaluación de la sustentabilidad mediante el uso de indicadores, con el soporte de una base de datos estructurada y actualizada que permita consultar y visualizar diferentes escenarios.

El desempeño de las fincas y la capacidad de gestión de los agricultores dependen de los cultivos/finanzas y la tecnología empleada; para conocer el grado de sustentabilidad de las fincas es necesario como punto de partida, evaluar los cultivos prevalentes que maneja el agricultor.

Los resultados de la evaluación de la sustentabilidad de las fincas con cultivos prevalentes, alerta a los responsables de las políticas agrarias a implementar enmiendas y correcciones a los planes y programas del estado en el tratamiento de la pequeña y mediana agricultura nacional.

Los resultados obtenidos en esta investigación son de mucha utilidad para continuar con el estudio del diseño y modelamiento de los sistemas agrícolas sustentables.

\section{MATERIALES Y MÉTODOS}

La investigación se realizó en la cuenca media y baja del río Supe, jurisdicción del distrito Supe, provincia de Barranca, departamento de Lima; es de tipo mixto (cualitativo y cuantitativo), transversal, descriptivo y no experimental.

Los materiales utilizados en la investigación fueron: Cartografía digital de la cuenca del río Supe, fichas de encuesta, GPS, muestreador de suelos, penetrómetro y un altímetro. Además los softwares como ArcGis V 10.1, SPSS v20, Microsoft Word, Excel y Auto-CAD.

La metodología utilizada consistió en la realización secuencial de las siguientes actividades:

a) Caracterización de los sistemas prediales, que consistió en la descripción analítica de las unidades de producción agropecuaria. Se aplicó técnicas de recolección y análisis de datos con el fin de obtener y procesar la información; b) levantamiento de información de las fincas con cultivos prevalentes, actividad que tuvo por finalidad recoger información de los agricultores en base a encuestas, información referida a componentes agro-ecológicos, técnico-productivos y socio-económicos que tienen mayor influencia en el desempeño de los sistemas productivos locales; c) ordenamiento y análisis de la información obtenida de diversas fuentes, de acuerdo a variables seleccionadas utilizando para ello técnicas de la estadística descriptiva; d) descripción de las características prediales como resultado del análisis previo de las variables de mayor interés y luego de un proceso inductivo que culminó en la integración de los componentes, se describen las características más importantes de los predios, indican- 
do que los componentes agro-ecológicos describen los factores naturales, el estado de los agroecosistemas en relación al suelo, plagas, climáticos y otros, la diversidad vegetal existente en las fincas, los problemas ecológicos de la zona. Los componentes técnico - productivos describen: El uso de la tierra, los cultivos prevalentes, las tecnologías y prácticas campesinas, sistemas de cultivo, sistemas de producción. Los componentes socio-económicos analizan: Los recursos de tierra, capital y trabajo, la rentabilidad de los cultivos, las condiciones de vida de los productores y los niveles de organización campesina; e) La evaluación de la sustentabilidad de fincas consistió en la evaluación de fincas con cultivos prevalentes en los cuales se midió la sustentabilidad aplicando indicadores económicos, ecológicos y socioculturales integrándolos mediante el análisis tipo multicriterio, así como indicadores de calidad del suelo.

Se ha empleado procedimientos participativos con los agricultores para la evaluación de las fincas seleccionadas. La tabla 1 muestra los indicadores y subindicadores utilizados para evaluar la sustentabilidad en el presente estudio.

Tabla 1. Indicadores y subindicadores de sustentabilidad empleando la metodología «tipo multicriterio»

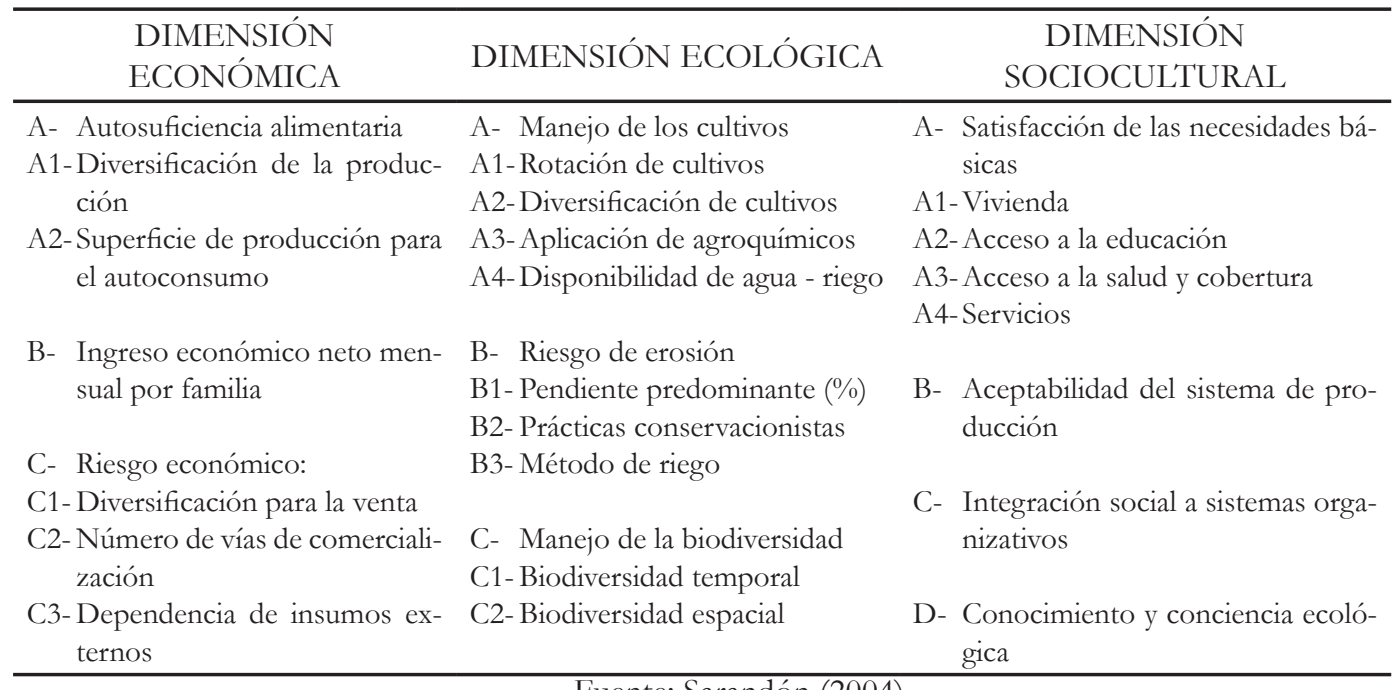

Fuente: Sarandón (2004)

Descripción y ponderación de los indicadores elegidos. Según el análisis de las condiciones de la zona y de acuerdo al grado de conocimiento del investigador se hizo algunas modificaciones a la metodología planteada por Sarandón et al. (2004), referidas a la descripción de subindicadores y sus unidades de medida y al peso de los indicadores, lo cual se refleja en las fórmulas empleadas.

La información para cada finca se obtuvo mediante entrevistas a los propietarios y observación de campo. Para cada indicador y subindicador se ha consignado un valor entre 0 a 4 . A continuación se presenta las fórmulas para las tres dimensiones analizadas:

Indicador Económico $(\mathrm{IK})=\{2[(\mathrm{~A} 1+\mathrm{A} 2) / 2]+2 \mathrm{~B}+[(\mathrm{C} 1+\mathrm{C} 2+\mathrm{C} 3) / 3]\} / 5$

Indicador Ecológico $(\mathrm{IE})=\{(2 * \mathrm{~A} 1+\mathrm{A} 2+\mathrm{A} 3) / 4+(2 \mathrm{~B} 1+\mathrm{B} 2+\mathrm{B} 3) / 4+(\mathrm{C} 1+\mathrm{C} 2) / 2\} / 3$

Indicador Sociocultural (ISC) $=\{2[(2 * A 1+2 \mathrm{~A} 2+\mathrm{A} 3+2 * \mathrm{~A} 4) / 7]+2 \mathrm{~B}+\mathrm{C}+\mathrm{D}\} / 6$ 
Con los datos de los macroindicadores económicos (IK), ecológicos (IE) y socioculturales (ISC), se calcula el índice de sustentabilidad general (IS-G), que valora las tres dimensiones por igual:

$$
\mathrm{IS}-\mathrm{G}=(\mathrm{IK}+\mathrm{IE}+\mathrm{ISC}) / 3
$$

El segundo método utilizado fue una adaptación al «método agroecológico rápido para la evaluación de la sostenibilidad de cafetales» (Altieri y Nicholls, 2002), que utiliza criterios de calidad de suelo y salud del cultivo, con modificaciones de adaptación a la cuenca, a los cultivos prevalentes y calificando la calidad del suelo.

Se seleccionaron diez indicadores de calidad de suelo; cada indicador se estima en forma separada y se le asigna un valor de 1 a 10 (siendo 1 el valor menos deseable, 5 un valor medio y 10 el valor deseado) de acuerdo a las características que presenta el suelo o el cultivo, y los atributos a evaluar para cada indicador.

\section{RESULTADOS}

\section{Caracterización geoespacial del territorio}

Se determinó las características fisiográficas, morfológicas y ecológicas del área estudiada, usando las imágenes satelitales LandSat, SRTM y aerofotografías, apoyadas con cartografía e información digitalizada; identificando primero el límite de la cuenca baja y media del río Supe (Ayora, 2015).

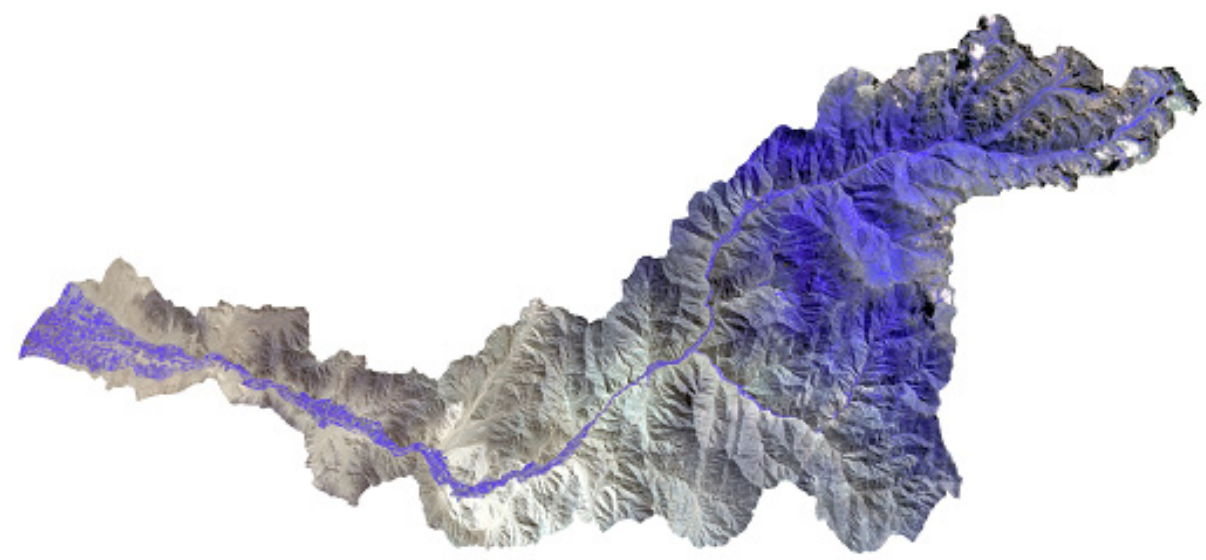

Figura 1. Imagen satelital de la cuenca del río Supe

Determinación de atributos y evaluación de la sustentabilidad de parcelas

Las unidades de análisis fueron las fincas que albergan cultivos prevalentes.

Atributos de los sistemas prediales. Mediante encuestas y talleres participativos, se obtuvo información de las fincas y de sus conductores, referentes a: sexo, edad del jefe de familia, nivel de instrucción del responsable de la parcela, provisión de servicios básicos, ingreso mensual, crianza de animales, medio de comunicación, participación en organizaciones, propiedad de la tierra y superficie que conduce. 
Tabla 2. Características principales de las fincas

\begin{tabular}{|c|c|c|c|c|c|c|c|c|c|c|c|}
\hline $\begin{array}{l}\text { Nivel } \\
\text { instruc }\end{array}$ & $(\%)$ & $\begin{array}{l}\text { Ingreso } \\
(\mathrm{S} .)\end{array}$ & $(\%)$ & $\begin{array}{l}\text { Tít } \\
\text { prop } \\
\text { Sí (\%) }\end{array}$ & $\begin{array}{l}\text { tulo } \\
\text { siedad } \\
\text { No }(\%)\end{array}$ & $\begin{array}{l}\text { Venta de } \\
\text { producción }\end{array}$ & $(\%)$ & $\begin{array}{l}\text { Actividad } \\
\text { complemt }\end{array}$ & $(\%)$ & $\begin{array}{l}\text { Edad } \\
\text { años }\end{array}$ & $(\%)$ \\
\hline Ninguno & 3,1 & $<=400$ & 1,0 & 63,3 & 36,7 & Intermed & 13,3 & Turismo & 2,0 & $<=28$ & 4,1 \\
\hline Primaria & 48,0 & $401-660$ & 29,6 & & & Mayoristas & 71,4 & Agricultura & 83,7 & $29-30$ & 12,2 \\
\hline Secundaria & 45,5 & $661-920$ & 62,2 & & & Minorista & 4,1 & Ganadería & 4,1 & $41-52$ & 23,5 \\
\hline \multirow[t]{2}{*}{ Técnica } & 3,1 & $921-1,180$ & 3,1 & & & Otros & 11,2 & Comercio & 5,1 & $53-64$ & 32,7 \\
\hline & & 1,181 - & & & & & & & & & \\
\hline \multirow[t]{3}{*}{ Total } & 100,0 & 1,440 & 1,0 & & & Total & 100,0 & Otras & 5,1 & $65-76$ & 23,5 \\
\hline & & $>1,441$ & 3,1 & & & & & Total & 100,0 & $77+$ & 4,1 \\
\hline & & Total & 100,0 & & & & & & & Total & 100,0 \\
\hline
\end{tabular}

Fuente: Investigador - encuestas

Como se observa en la tabla 2 , la propiedad y conducción de los predios están en manos de las primeras generaciones de migrantes; población adulta (el 83,76 \% de ellos tienen edades mayores a 41 años) con bajo nivel de escolaridad. El nivel de instrucción se distribuye en 48,0\% para el nivel primario, un 45.9\% de agricultores tienen estudios secundarios completos; y solo un 3,1\% de encuestados habían efectuado estudios de nivel técnico. Escenario que denota la escasez de técnicos en el área intervenida.

Tabla 3. Características de las fincas

\begin{tabular}{|c|c|c|c|c|c|c|c|c|c|c|c|}
\hline \multirow{2}{*}{$\begin{array}{c}\begin{array}{c}\text { Vivienda } \\
\text { agricult }\end{array} \\
\begin{array}{c}\text { Material } \\
\text { noble }\end{array}\end{array}$} & \multirow{2}{*}{$\frac{(\%)}{9,2}$} & \multicolumn{2}{|c|}{$\begin{array}{l}\text { Energía eléctrica } \\
\text { Sí }(\%) \text { No }(\%)\end{array}$} & \multicolumn{2}{|c|}{$\begin{array}{l}\text { Agua potable } \\
\text { Sí }(\%) \text { No }(\%)\end{array}$} & \multicolumn{2}{|c|}{$\begin{array}{l}\text { Agua de riego } \\
\text { Sí }(\%) \text { No }(\%)\end{array}$} & \multicolumn{2}{|c|}{$\begin{array}{c}\text { Aplicada } \\
\text { Mt-orgánica } \\
\text { Si }(\%) \text { No (\%) }\end{array}$} & \multirow{2}{*}{$\begin{array}{l}\text { Asociado } \\
\text { Regantes }\end{array}$} & \multirow{2}{*}{$\begin{array}{r}(\%) \\
83,7\end{array}$} \\
\hline & & 89,8 & 10,2 & 48,0 & 52,0 & 68,4 & 31,6 & 27,6 & 72,4 & & \\
\hline Adobe calami & 73,5 & & & & & & & & & Productores & 10,2 \\
\hline Caña y barro & 17,3 & & & & & & & & & Deportiva & 2,0 \\
\hline \multirow[t]{2}{*}{ Total } & 100,0 & & & & & & & & & Religiosa & 4,1 \\
\hline & & & & & & & & & & Total & 100,0 \\
\hline
\end{tabular}

Fuente: Investigador - encuestas

En la tabla 3 se observa que el tipo de vivienda característica es de adobe y calamina (73,5\%); solo el $48 \%$ de la población consume agua potabilizada; el 89,8\% dispone de energía eléctrica. En la zona aún no se ha resuelto el problema de la propiedad de la tierra, pues solo el 63,3\% de los agricultores tienen título de propiedad.

Los cultivos prevalentes de la zona son: el maíz duro amarillo (Zea mays), la caña de azúcar (Saccharum officinarum), el ají páprika (Capsicum annuum L.); y frutales como el maracuyá (Passiflora Edulis) y palto (Persea americana). En la tabla 2 se detalla la extensión cultivada de los cultivos prevalentes, así como los rendimientos promedio de estos en la zona estudiada. 
El tamaño promedio de la finca o parcela es de 3,53 ha. Las áreas son cultivables en su totalidad, con suelos aptos para la agricultura intensiva, con suministro de agua para riego por turnos. El agricultor opta por un cultivo prevalente pero en algunos casos conduce más de uno en la finca bajo una estrategia de diversidad, para atenuar impactos económicos o biológicos. Se confirma la presencia mayoritaria de pequeños productores en toda la zona.

Tabla 4. Ingresos económicos familiares

\begin{tabular}{cccccc}
\hline $\begin{array}{c}\text { Sector de la } \\
\text { Cuenca }\end{array}$ & $\begin{array}{c}\text { Total utilidad neta } \\
\text { anual familiar (S/.) }\end{array}$ & $\begin{array}{c}\text { Total utilidad neta } \\
\text { mensual familiar (S/.) }\end{array}$ & $\begin{array}{c}\mathrm{N}^{\circ} \text { de personas que } \\
\text { dependen de la finca }\end{array}$ & $\begin{array}{c}\text { Ingreso mensual } \\
\text { per cápita (S/.) }\end{array}$ & $\begin{array}{c}\text { Ingreso mensual } \\
\text { per cápita (\$) }\end{array}$ \\
\hline Baja & 8395.2 & 699.6 & 2.75 & 254.40 & 80.51 \\
Media & 5963.1 & 496.9 & 2.83 & 175.59 & 55.57 \\
\hline
\end{tabular}

Fuente: Encuesta del Investigador

La tabla 4 muestra que la economía de los agricultores es crítica; el ingreso mensual per cápita en la cuenca baja es $\$ 80,51$, y en la cuenca media $\$ 55,57$; estos valores son bajos de acuerdo a los estándares internacionales. Se verifica de este modo un estado de pobreza en la zona rural de las Cuencas Baja y Media.

El ingreso mensual promedio para la cuenca baja es S/. 755,36; el ingreso mensual promedio para la cuenca media es S/.747,59; y de toda la zona en estudio es S/. 753,06

De los 98 jefes de familia encuestados, el $83.7 \%$ se dedica completamente a la agricultura y el 16,3\% además de la agricultura tiene otras actividades complementarias.

Los sistemas de subsistencia en conjunto representan el 4,5\% y los sistemas semiintensivos para la venta suman el 95,5\% de prevalencia en la zona. La variable que define ambos grupos de sistemas es la conexión de la producción agropecuaria con el mercado. Para este estudio los sistemas de subsistencia están agrupados en: agricultura de subsistencia (1,25\%), crianzas menores extensivas (3,25\%). Los sistemas para la venta son: cultivos perennes intensivos, como el espárrago y la caña de azúcar (16,65\%), cultivos intensivos transitorios (59,30\%), fruticultura semiintensiva (19,55\%).

Evaluación de la sustentabilidad de fincas. Para evaluar la sustentabilidad de fincas en este estudio, se aplicó dos metodologías: el análisis «tipo multicriterio» (Sarandón et al., 2004) y el «método agroecológico rápido para la evaluación de la sostenibilidad de cafetales» utilizando solo criterios de calidad de suelo (Altieri y Nicholls, 2002).

Evaluación de fincas utilizando el análisis multicriterio. Se evaluó 18 fincas (tres fincas por cada cultivo prevalente), en las cuales se aplicó indicadores de sustentabilidad (Sarandón et al., 2004), calificando de acuerdo al tipo de indicador (económico, ecológico y sociocultural), en una escala de 0 a 4 . Para determinar la sustentabilidad se aplicó las fórmulas referidas en la metodología; se tomó como referencia el valor mínimo de 2.0, debajo del cual se considera como no sostenible. 
Tabla 5. Evaluación de la sustentabilidad de fincas con cultivos prevalentes

\begin{tabular}{cccccccccccccccc}
\hline Finca & $\begin{array}{c}\text { AA } \\
(2)\end{array}$ & $\begin{array}{c}\text { IM } \\
(2)\end{array}$ & RE & IK & MC & RE & MB & IE & $\begin{array}{c}\text { SN } \\
(2)\end{array}$ & $\begin{array}{c}\text { Ac } \\
(2)\end{array}$ & IS & CE & ISC & IS-C & Sustent \\
\hline Maíz A D & 1.33 & 2.67 & 1.89 & 2.0 & 2.87 & 3.75 & 2.67 & 3.09 & 2.90 & 3.00 & 3.33 & 2.33 & 2.91 & 2.66 & SI \\
Palto & 2.00 & 2.67 & 2.68 & 2.42 & 2.67 & 3.58 & 2.50 & 2.92 & 3.00 & 2.00 & 2.67 & 3.00 & 2.61 & 2.65 & SI \\
Caña A & 2.17 & 3.33 & 2.11 & 2.64 & 3.07 & 3.92 & 2.50 & 3.16 & 2.62 & 3.00 & 3.00 & 2.33 & 2.76 & 2.86 & SI \\
Maíz M & 1.83 & 2.67 & 2.33 & 1.35 & 3.13 & 3.00 & 3.17 & 3.1 & 2.81 & 2.33 & 2.67 & 2.67 & 2.6 & 2.75 & NO \\
Maracuyá & 1.33 & 3.00 & 2.22 & 1.29 & 2.47 & 3.83 & 2.33 & 2.88 & 3.05 & 3.33 & 3.67 & 2.67 & 3.18 & 2.74 & NO \\
Páprika & 1.00 & 2.65 & 1.44 & 1.5 & 1.35 & 2.85 & 1.15 & 2.0 & 2.25 & 1.67 & 2.00 & 2.67 & 2.5 & 1.98 & NO \\
\hline \multicolumn{7}{c}{ Nota: Valores promedio iguales o menoeres a 2.00 indican sistemas no sostenibles. }
\end{tabular}

Leyenda:

$\mathrm{IK}=$ indicador económico: $\mathrm{AA}=$ autosuficiencia alimentaria; $\mathrm{IM}=$ ingreso económico neto mensual por familia; $\mathrm{RE}=$ riesgo económico

$\mathrm{IE}=$ indicador ecológico: $\mathrm{MC}=$ manejo de cultivo; $\mathrm{RE}=$ riesgo de erosión; $\mathrm{MB}=$ manejo de la biodiversidad.

ISC = indicador sociocultural: SN satisfacción de las necesidades básicas; Ac= aceptabilidad del sistema;

IS = integración social;

$\mathrm{CE}=$ conocimiento y conciencia ecológica.

IS-G = índice de sustentabilidad general.

\section{Evaluación de la sustentabilidad de las fincas utilizando criterios de calidad del suelo}

Se evaluaron otras 18 parcelas (3 parcelas por cada cultivo) aplicando indicadores prácticos que estiman la calidad del suelo, con la participación del agricultor se calificó con una escala de 1 a 10 para cada indicador, luego se obtuvo los promedios para cada cultivo. Para estimar la sustentabilidad se tomó como referencia el valor mínimo de 5, debajo del cual se considera como no sostenible al sistema evaluado.

Tabla 6. Evaluación de sustentabilidad, usando criterios de calidad del suelo

\begin{tabular}{llcccccc}
\hline & CALIDAD DEL SUELO & Maíz-A & Palto & Caña & Maíz M & Maracuyá & Ají páprika \\
\hline 1 & Estructura y textura & 6.7 & 6.7 & 5.3 & 6.7 & 8.3 & 3.7 \\
2 & Compactación e infiltración & 6.7 & 8.3 & 6.7 & 8.3 & 8.3 & 3.7 \\
3 & Profundidad del suelo & 10.0 & 10.0 & 10.0 & 6.7 & 8.3 & 8.3 \\
4 & Estado de residuos & 3.7 & 3.7 & 5.0 & 6.7 & 5.0 & 3.7 \\
5 & Materia orgánica, color y color & 3.7 & 5.0 & 5.0 & 5.0 & 3.7 & 3.7 \\
6 & Retención de humedad & 8.3 & 8.3 & 8.3 & 3.7 & 8.3 & 2.3 \\
7 & Desarrollo de raíces & 6.7 & 10.0 & 10.0 & 5.0 & 8.3 & 3.7 \\
8 & Cobertura del suelo & 1.0 & 3.7 & 5.0 & 3.7 & 6.7 & 1.0 \\
9 & Erosión & 10.0 & 10.0 & 10.0 & 6.7 & 8.3 & 6.7 \\
10 & Actividad biológica & 2.3 & 6.7 & 2.3 & 3.7 & 3.7 & 2.3 \\
& PROMEDIO & 5.9 & 7.2 & 6.8 & 5.6 & 6.9 & 3.9 \\
\hline
\end{tabular}

Fuente: Elaboración en base a evaluación en campo de fincas seleccionadas. 
Construcción de diagramas tipo «ameba». Con los valores obtenidos (tabla 3) se construyó diagramas tipo ameba o «tela de araña» que permiten visualizar el estado general de la calidad del suelo, precisando que mientras más se aproxime la ameba del cultivo al diámetro mayor del círculo (valor 10) el sistema es más sostenible.
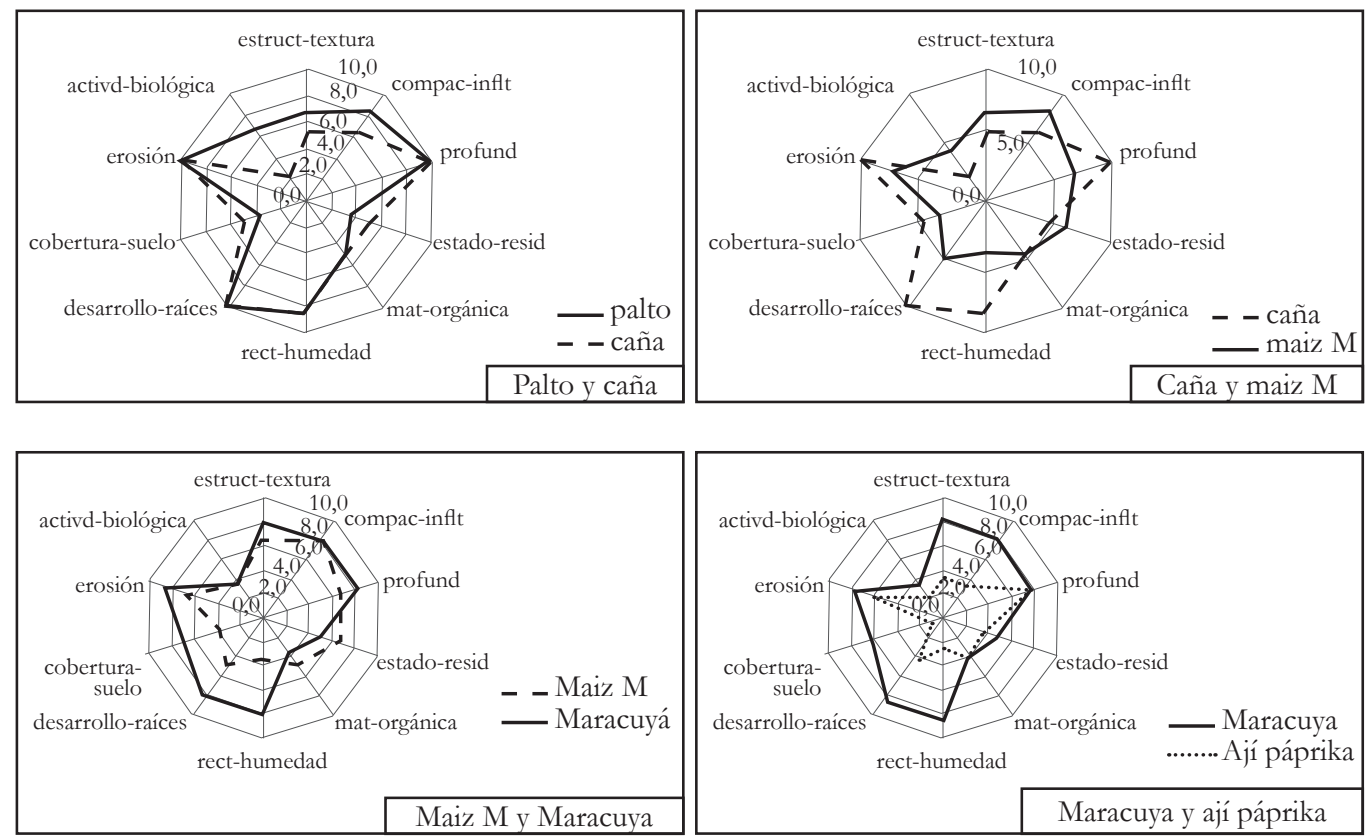

Figura 2. Diagramas tipo ameba

\section{DISCUSIÓN}

Los resultados corroboran la afirmación de que la caracterización detallada de los aspectos biofísicos, socioeconómicos y culturales de un territorio permiten evaluar la sustentabilidad del área a intervenir (Merma, 2012). En el presente estudio, se incorporó la característica de fincas con cultivos prevalentes.

La insustentabilidad de las fincas con cultivos de maíz morado, ají páprika y maracuyá se debe al gran riesgo económico que encierran, además que no contribuyen a la autosuficiencia alimentaria de la zona. Se observa que estas fincas no alcanzan el umbral mínimo de sustentabilidad, resultando no sustentables; precisamos que para que una finca sea sustentable el valor de sustentabilidad de los indicadores económicos (IK), ecológicos (IE) y socioculturales (ISC) debe ser igual o mayor a dos (Sarandón et al., 2004).

En la estructura socio-económica de la zona se mantiene la coexistencia de dos tipos de lógicas de producción: fincas de racionalidad capitalista (cuenca baja) y fincas de racionalidad tradicional o de subsistencia (cuenca media). Los medios de producción (tierra, mano de obra, bienes de capital) son utilizados por el agricultor de acuerdo a su ubicación geográfica (Malagón y Praguer, 2001). 
Los sistemas de subsistencia y los sistemas semiintensivos para la venta suman el 95,5\% de prevalencia en la zona. La variable que define ambos grupos de sistemas es la conexión de la producción agropecuaria con el mercado. Para este estudio los sistemas de subsistencia están agrupados en: agricultura de subsistencia y crianzas menores extensivas. Los sistemas para la venta son: cultivos perennes intensivos, como el espárrago y la caña de azúcar que representan el 16,65\%, cultivos intensivos transitorios que alcanzan un 59,30\% y, fruticultura semiintensiva en 19,55\%.

Los resultados de la evaluación de la sustentabilidad, utilizando criterios de calidad del suelo, demuestran que los cultivos de palto y maracuyá tienen suelos de mejor calidad ecológica, lo que significa una mayor sostenibilidad en relación suelo-planta. En cambio, el cultivo de páprika tiene promedio menor a 5,0 que demuestra que ocupa suelos con limitaciones edáficas que los vuelven poco sostenibles.

Comparando estos resultados con los obtenidos al evaluar la sustentabilidad por el método multicriterio, se advierte que solamente coinciden en las fincas con ají páprika. Esta variación se explica en la medida que en la segunda evaluación solo se usa un subindicador puntual que es la calidad del medio físico (suelo), en contraste con la evaluación multicriterio que integra las dimensiones ecológicas, económicas y socioculturales.

En los diagramas del tipo ameba, se corrobora gráficamente que los cultivos con mayor grado de sustentabilidad son: El maíz amarillo duro, el palto y la caña de azúcar, y quedan los cultivos de ají páprika, maíz morado y maracuyá como insustentables.

\section{CONCLUSIONES}

La determinación de los atributos técnicos productivos, socioculturales, así como de las características agropecuarias y económicas de las fincas con cultivos prevalentes, sirvió para evaluar su sustentabilidad.

En la estructura socio-económica de la zona se mantiene la coexistencia de dos tipos de lógicas de producción: fincas de racionalidad capitalista (cuenca baja) y fincas de racionalidad tradicional o de subsistencia (cuenca media). Los medios de producción (tierra, mano de obra, bienes de capital) son utilizados por el agricultor de acuerdo a la ubicación geográfica.

Las metodologías de análisis multicriterio, y de calidad del suelo, son adecuadas para evaluar la sustentabilidad de las fincas con cultivos prevalentes en el ámbito de estudio.

Los diagramas tipo ameba, son de utilidad para mostrar gráficamente el nivel de sustentabilidad de las fincas evaluadas, así como para efectuar las comparaciones entre los cultivos evaluados y el grado óptimo de sustentabilidad.

Los cultivos prevalentes como el maíz amarillo duro, la caña de azúcar y el palto son los que resultan sustentables en la cuenca media y baja del río Supe. 
De los cultivos prevalentes evaluados, solo la mitad alcanza sustentabilidad; por lo que se requiere de un modelado del funcionamiento de estas fincas para analizar su comportamiento y proponer mejoras o enmiendas tendientes a mejorar su desempeño y, propender a la sustentabilidad.

\section{AGRADECIMIENTOS}

Al Ph.D. Absalón Vásquez Villanueva y la Ing. Lisbeth Ayora Roque.

\section{REFERENCIAS BIBLIOGRÁFICAS}

Altieri, Miguel y Nicholls, Clara. 2002. Agroecología: teoría y práctica para una agricultura sustentable. Serie Textos Básicos. México: ONU-PNUMA.

Ayora, Lorenzo. 2015. «Caracterización geoespacial de la cuenca media y baja del río Supe para identificar sistemas agrícolas prevalentes». Revista Académica UNASAM. Vol. 2. Huaraz. 39-65.

Malagón, Ricardo y Prager, Martín. 2001. El enfoque de sistemas: Una opción para el análisis de las unidades de producción agrícola. Bogotá: Universidad Nacional de Colombia.

Merma, Isaías. 2012. Evaluación y diseño de fincas en selva alta bajo sistemas de cultivos prevalentes, en la Convención- Cusco. Tesis de PhD. Agricultura Sustentable, Universidad Nacional Agraria La Molina. Lima: UNALM.

Sarandón, Santiago. 2004. El desarrollo y uso de indicadores para evaluar la sustentabilidad de los agroecosistemas en agroecología: El camino hacia una agricultura sustentable. La Plata: Ediciones Científicas Americanas.

Fecha de recepción: 06 de noviembre 2015

Fecha de aceptación: 02 de diciembre 2015

\section{Correspondencia}

Lorenzo Ayora Garagate

lorenzoayora@hotmail.com 


\section{FOTOGRAFÍAS}

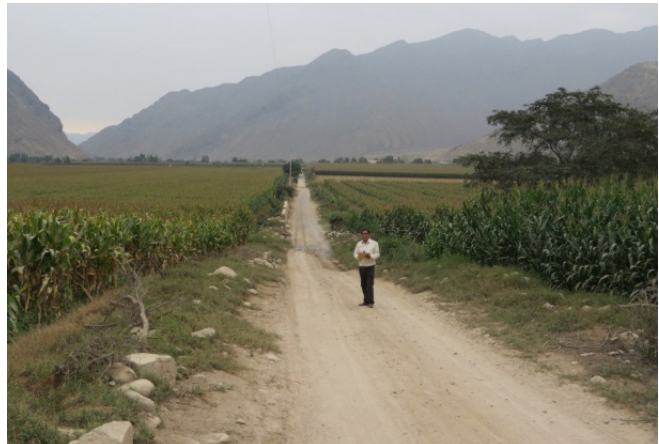

Cultivo de maíz amarillo - Cuenca media

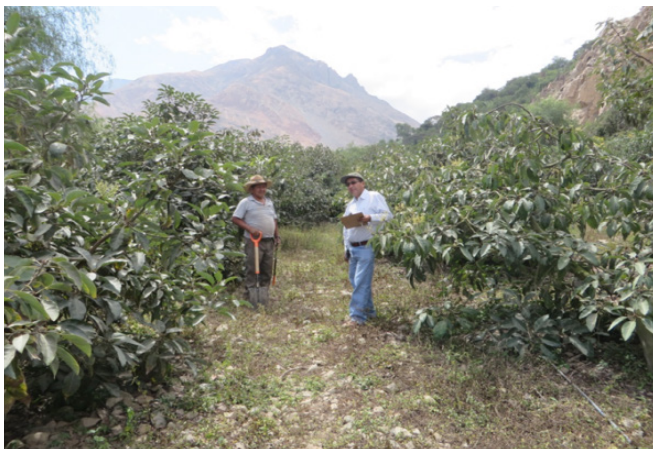

Cultivo de palto - Cuenca media

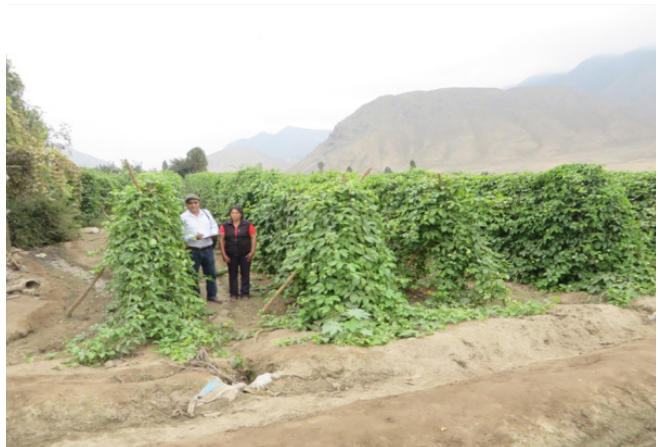

Cultivo de maracuyá - Cuenca baja

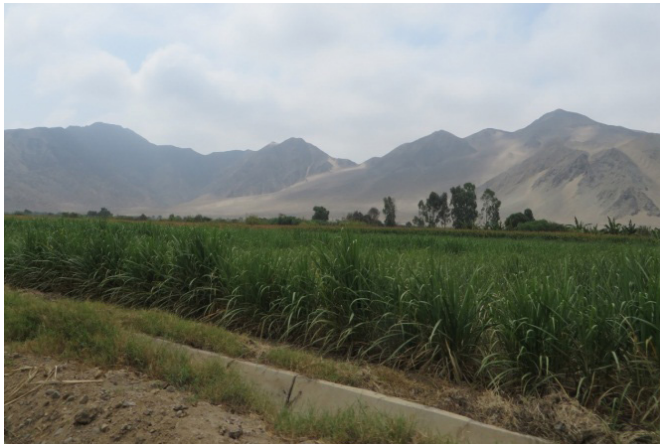

Cultivo de caña de azúcar - Cuenca baja

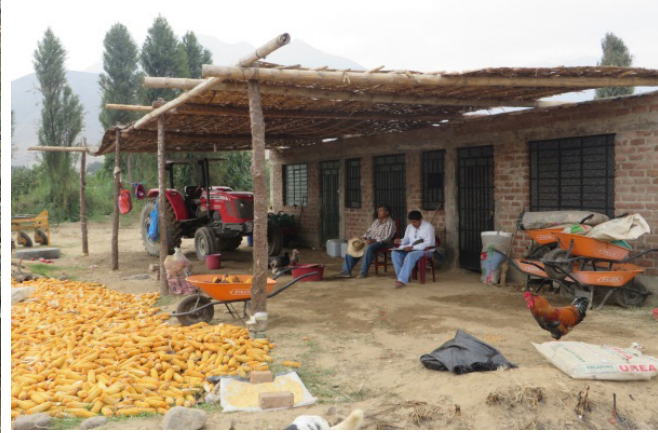

Modelo de finca sustentable - Cuenca media

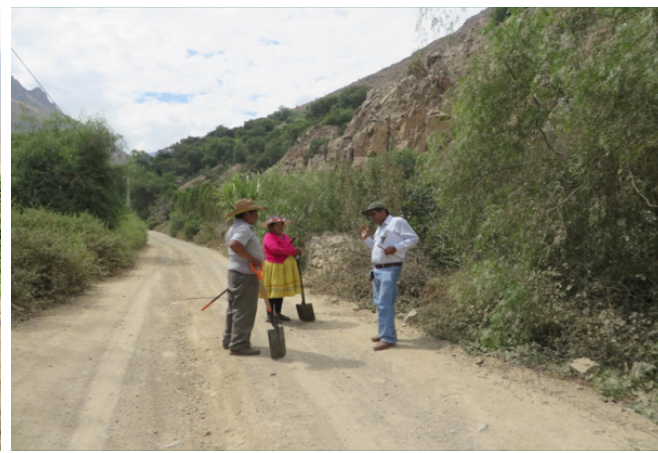

Entrevista con agricultores - Cuenca media 